\title{
Gas-Phase Cationization and Protonation of Neutrals Generated by Matrix-Assisted Laser Desorption
}

\author{
Bing H. Wang, Klaus Dreisewerd, Ute Bahr, Michael Karas, and \\ Franz Hillenkamp \\ Institute of Medical Physics and Biophysics, University of Muenster, Muenster, Germany
}

The ionization mechanisms involved in matrix-assisted ultraviolet laser desorption/ionization (MALDI) were studied with a time-of-flight mass spectrometer. When protonated or cationized quasimolecular ions generated by MALDI are not extracted promptly, their abundance is a function of the delay time between laser irradiation and ion extraction, maximizing at an optimum delay time (DTM) of a few hundred nanoseconds. The ion abundance at DTM exceeds that of prompt extraction by a factor of 2 or more. Increasing the cation density near the sample surface reduces the DTM, whereas increasing the desorption laser irradiance has the opposite effect. The enhancement suggests extensive gas-phase ion-molecule reactions after irradiation by the desorption laser has ceased. (J Am Soc Mass Spectrom 1993, 4, 393-398)

W ith its versatility and high sensitivity, matrix-assisted laser desorption/ionization (MALDI) represents one of the most important developments in ionization technique for large molecules in recent years [1]. Proteins in excess of 200 $\mathrm{kDa}$ can now be ionized [2], and other important classes of compounds, such as polynucleotides [3], oligosaccharides [4, 5], and synthetic polymers [6], have also been shown to be amenable to this technique. A detection limit of femtomoles has been demonstrated when MALDI is coupled to a time-offlight (TOF) mass spectrometer $[7,8]$. The utility of MALDI makes a thorough understanding of the mechanisms involved in the technique particularly desirable.

A key feature of MALDI is the mixing of an analyte with a small organic compound that absorbs resonantly at the laser wavelength used for desorption. It has been observed that although MALDI generates predominantly cationized quasimolecular ions for some peptides, it produces predominantly protonated quasimolecular ions for other peptides and probably all proteins [9]. Currently, it is still unclear how the absorption of photons by the matrix molecules leads to the desorption of large molecules. The role of the matrix molecules in the cationization as well as the protonation process is also poorly understood.

\footnotetext{
*Dr. Wang's present address is Department of Chemistry, Massachusetts Institute of Technology, Cambridge, MA 02139.

Address reprint requests to Klaus Dreisewerd. Institute of Medical Physics and Biophysics, University of Muenster, Muenster, Germany.
}

Earlier studies have shown that cationized quasimolecular ions produced by laser desorption/ ionization (LDI) without matrix are the result of ion-molecule reactions in the gas phase [10-12]. For the formation of protonated molecular ions by LDI, Parker and Hercules [13] suggested that "pair production" is the major mechanism, based on the result of the study of deuterated amino acids.

Previous work from this laboratory has shown that under certain experimental conditions, LDI produces more radical molecular ions than protonated ones [14]. Tryptophan, for instance, usually gives abundant $[\mathrm{M}+\mathrm{H}]^{+}$but little $\mathrm{M}^{+}$; however, when the sample was cooled to $90 \mathrm{~K}$, the abundances of $\mathrm{M}^{+}$and $[\mathrm{M}+$ $\mathrm{H}^{+}$were reversed. Furthermore, when ions were not extracted promptly, multiple hydrogen attachment to the molecules was observed. Speir et al. [15] also showed recently that neutral moieties generated by LDI can react with ions trapped in an ion cyclotron resonance cell in the gas phase. All of these observations seem to suggest that in $\mathrm{LDI}_{f}[\mathrm{M}+\mathrm{H}]^{+}$is the product of gas-phase reactions between $\mathrm{M}^{+}$and desorbed molecules.

Consistent with the above observations, another recent study in this laboratory showed that a host of organic compounds, which can assist the desorption/ ionization of proteins, form odd-electron molecular ions [16]. The study consequently suggests that in MALDI, the $[\mathrm{M}+\mathrm{H}]^{+}$ion of the analyte is the product of gas-phase reactions between matrix ions and analyte molecules, with photoradical matrix ions initiating the reactions. 
To obtain more evidence that ion-molecule reactions in the gas phase may account for the ionization of analyte molecules in MALDI, the effect of delaying the extraction of ions was studied. Delaying the extraction presumably gives more time for bimolecular reactions or other processes to take place in the ion source [17].

\section{Experimental}

Figure 1 shows the experimental setup used in this study. A $\mathrm{KrF}$ laser (laser I) with a wavelength of 248 $\mathrm{nm}$ and a pulse width of $15 \mathrm{~ns}$ was used for desorption. The laser was attenuated and focused to approximately $50 \mu \mathrm{m}$ to give an irradiance of $3 \times 10^{6} \mathrm{~W} / \mathrm{cm}^{2}$. Under prompt extraction conditions, the sample was held at a potential of $3 \mathrm{kV}$, and the counterelectrode of the extraction optics, $5 \mathrm{~mm}$ in front of the sample probe, was held at ground potential. For delayed extraction, this counterelectrode is initially floated at 3 $\mathrm{kV}$ and switched to ground within $80 \mathrm{~ns}(90-10 \%$ value) after a variable delay time. In the cross-beam experiments, in addition to laser $\mathrm{I}$, another $\mathrm{KrF}$ excimer laser (laser II, wavelength $248 \mathrm{~nm}$, pulse width $20 \mathrm{~ns}$ ) was used to irradiate a neat NaI sample to produce a plume of $\mathrm{Na}^{+}$ions before laser I was fired. The $\mathrm{NaI}$ sample was placed on a glass surface mounted at a right angle to the main sample surface at a distance approximately $2 \mathrm{~mm}$ from the desorption area. Laser II was attenuated and focused to a spot approximately $200 \mu \mathrm{m}$ (horizontal) $\times 400 \mathrm{~m}$ (vertical). The irradiance was adjusted to approximately $4 \times 10^{7}$ $\mathrm{W} / \mathrm{cm}^{2}$. This second laser beam propagated parallel to the main sample surface, with the center of the beam approximately $250 \mu \mathrm{m}$ in front of this surface. The focusing lens has a focal length of $20 \mathrm{~cm}$. The timing of the two lasers, as well as the delay of the extraction pulse, was controlled by a master clock and a delay generator. The jitter in the timing for both laser emissions and the voltage switching was approximately 10 ns. Ions were detected in a TOF mass spectrometer equipped with a homebuilt single-stage ion reflector. The ion detector consists of a conversion dynode and a secondary electron multiplier. The voltage at the conversion dynode was $-10 \mathrm{kV}$ for detection of peptides with molecular masses below $2000 \mathrm{Da}$ and at $-18 \mathrm{kV}$ for detection of peptides with molecular masses above $200 \mathrm{Da}$. The signal from the electron multiplier was amplified and recorded by a transient recorder (LeCroy 9400); the digitized signal was then transferred to a Personal Computer for summation, storage, and display.

With the exception described below, samples were prepared by premixing $2 \mu \mathrm{L}$ of a peptide solution of appropriate concentration with $2 \mu \mathrm{L}$ of $5 \times 10^{-2} \mathrm{M}$ 2,5-dihydroxybenzoic acid (DHB) aqueous solution to give a mixture of the desired molar ratio of the peptide to DHB. Both solutions contained $10 \%$ ethanol. For

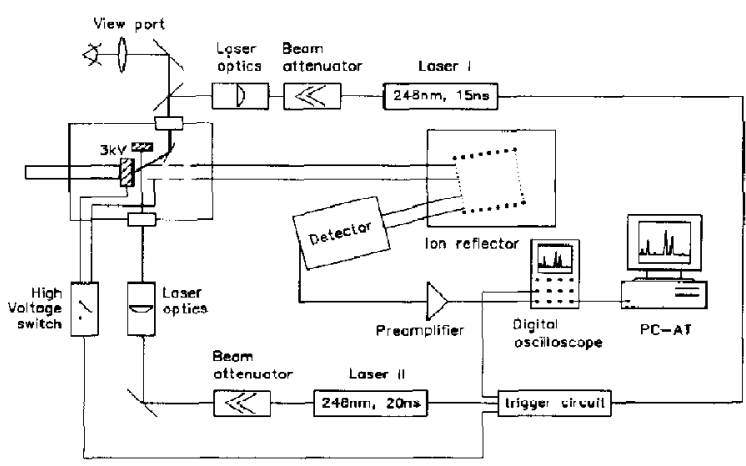

Figure 1. Schematic of the experimental setup used in the study. The system uses two KrF excimer lasers and an ion reflector. The timing circuit for triggering the lasers and the high-voltage switch are controlled by a master clock and a delay generator

[sar ${ }^{1}, \mathrm{val}^{5}$, ala ${ }^{8}$ ]-human angiotensin II (HATII) and melittin, the concentrations were $1 \times 10^{-4} \mathrm{M}$. For bovine insulin, the concentration was $3 \times 10^{-5} \mathrm{M}$; this solution contained $0.1 \%$ trifluoroacetic acid to enhance solubility. Typically, $1 \mu \mathrm{L}$ of the mixture solution was transferred to a silver substrate and dried in a stream of cool air. The diameter of the sample deposition on the substrate was approximately $2 \mathrm{~mm}$.

For the experiment. which required a wide variation in the molar ratio between DHB and gramicidin S, a $5 \times 10^{-2} \mathrm{M}$ DHB solution was mixed with appropriate amounts of gramicidin $S$ solution in the concentration range $5 \times 10^{-2}-7 \times 10^{-4} \mathrm{M} ; 0.5 \mu \mathrm{L}$ of these mixtures was transferred to the sample support. As a result, the total amount of DHB applied varied only by a factor of less than $2(18-33 \mathrm{nmol})$, and the full range of molar ratios of DHB:gramicidin $S$ varied from 0 (i.e., pure compound, no matrix) to 7680 . This precaution was taken to avoid possible influence of the applied $\mathrm{DHB}$ amount on the crystallization behavior on solvent evaporation. $\Lambda 10 \%$ ethanol aqueous solution was used as solvent for both DHB and gramicidin S, except for the two data points with pure gramicidim $S$ and a DHB to gramicidin $S$ molar ratio of 5:1. Pure cthanol was used in these cases to ensure complete solubility of gramicidin S.

Tor the cross-beam experiment, approximately $3 \mu \mathrm{L}$ of saturated NaI aqueous solution were applied to the glass surface and dried in a stream of warm air.

All peptide samples were purchased from Sigma Co. (Deisenhofen, Germany). DHB was purchased from Aldrich Chemical Co. (Steirheim, Germany). The compounds were used as purchased without further purification. All spectra are summed results of 20 singleshot spectra with the exception of HATII, where the summation was performed over 10 single-shot spectra. All intensities of ion signals were computed from the respective peak areas. 


\section{Results and Discussion}

\section{Gas-Phase Cationization Under MALDI Conditions}

Gramicidin S, a cyclic decapeptide, yields cationized quasimolecular ions readily by LDI; however, mixing gramicidin S with DHB lowers the threshold irradiance (the irradiance at which ions appear) for the observation of sodium- or potassium-attached quasimolecular ions. It also reduces the relative abundance of gramicidin $S$ fragment ions. Figure 2 shows the effect of varying the molar ratio of $D H B$ to gramicidin $S$ when the desorption irradiance was kept at $3 \times 10^{6} \mathrm{~W} / \mathrm{cm}^{2}$. As the molar ratio between DHB and gramicidin S varies from zero (neat gramicidin $S$ ) to $7600: 1$, the combined intensity of the $[\mathrm{M}+\mathrm{Na}]^{+}$and $[\mathrm{M}+\mathrm{K}]^{+}$ peaks varies, reaching a maximum at a ratio of 200:1.

To demonstrate that cationization may also occur through gas-phase cation attachment under matrix-assisted conditions, $\mathrm{Na}^{+}$was first produced by irradiating the NaI sample with laser II, followed by the desorption of gramicidin $\mathrm{S}$ with laser I after a delay of $100 \mathrm{~ns}$. After another delay of $200 \mathrm{~ns}$, the $3000-\mathrm{V}$ extraction pulse was applied. Figure 3a shows the mass spectrum of gramicidin $S$ resulting from the desorption of a gramicidin S/DHB mixture in a molar ratio of 1:2 when laser II was blocked. Figure $3 \mathrm{~b}$ shows the mass spectrum of the same sample when the block in front of laser II was removed. Signals from $\mathrm{Na}^{+}$, $\mathrm{Na}(\mathrm{NaL})^{+}$, and $\mathrm{Na}\left(\mathrm{NaI}_{2}^{+}\right.$dominate this spectrum, confirming that an abundance of $\mathrm{Na}^{+}$ions was generated by laser II. The intensity of the $\left[\mathrm{M}+\mathrm{Na}^{+}\right.$peak increased by a factor of 4 compared with that in Figure $3 a$, suggesting that cationization can occur in the gas phase under MALDI conditions. $\mathrm{Na}^{+}$was chosen in this experiment because it gives a higher yield of cationized quasimolecular ions of gramicidin $S$ than other alkali-metal ions under similar conditions. To ascertain that laser II did not cause direct desorption of gramicidin S, laser I was blocked. In this case only signals arising from the desorption of $\mathrm{NaI}$ were ob-

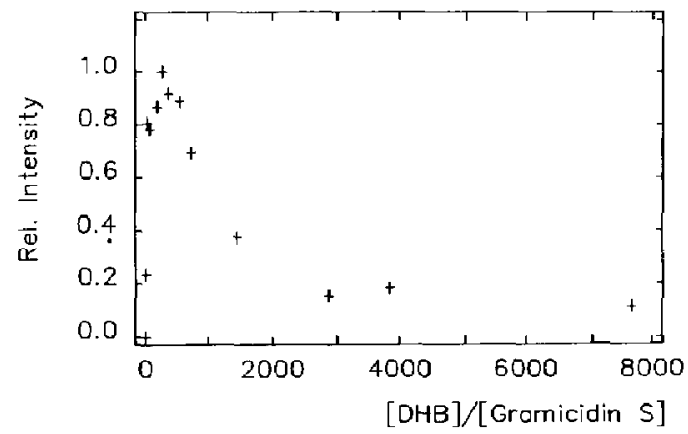

Figure 2. Combined intensity of $[\mathrm{M}+\mathrm{Na}]^{+}$and $[\mathrm{M}+\mathrm{K}]^{+}$ peaks of gramicidin $\mathbf{S}$ as a function of the molar ratio between DHB and gramicidin $S$. The desorption irradiance was approximately $3 \times 10^{6} \mathrm{~W} / \mathrm{cm}^{2}$. Ions were extracted promptly. served. In addition, when laser II was blocked after it had irradiated the NaI sample for some time, an enhancement in cationization was not observed. This is interpreted as proof that the enhancement was not caused by the deposition of sputtered $\mathrm{NaI}$ onto the main sample surface. Note that the $[\mathrm{M}+\mathrm{Na}]^{+}$peak in Figure $3 b$ is much broader than in Figure $3 a$, indicating that the $\mathrm{Na}^{+}$ions were spatially dispersed prior to reacting with gramicidin $S$ molecules, leading to the formation of a spatially dispersed $[\mathrm{M}+\mathrm{Na}]^{+}$cloud. This cloud of ions, when accelerated from the ion source, possesses a spread of kinetic energies that cause a dispersion in the ion flight time that cannot be fully corrected by an ion reflector. This cross-beam result shows that in an environment where both neutrals and ions quickly diffuse away, peptide molecules, like many small molecules $[18,19]$, can nevertheless react with other ions. It should be pointed out that although the cross-beam experiment has the advantage of separating desorption from cationization, thus allowing a better study of the matrix effect on both processes, it has the disadvantage of being very sensitive to the relative position of the two laser beams. This makes a systematic study based on this method difficult and rather time-consuming.

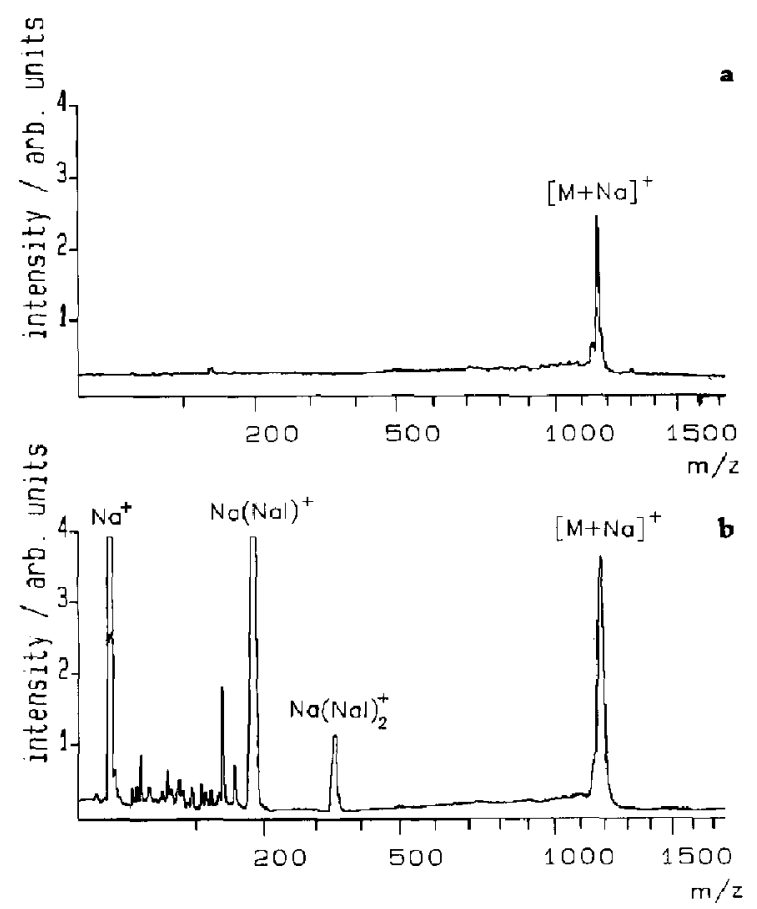

Figure 3. Mass spectra of gramicidin S: (a) "normal" desorption spectrum without additional $\mathrm{Na}^{+}$production by the second laser; (b) mass spectrum of gramicidin $S$ when the generation of a plume of $\mathrm{Na}^{+}$ions by a second laser preceded the desorption of gramicidin $\mathrm{S}$ by $100 \mathrm{~ns}$. The molar ratio between gramicidin $\mathrm{S}$ and DHB was $1: 300$. 
If gas-phase cation attachment is the dominating mechanism in the formation of cationized quasimolecular ions, then it should be possible to increase the abundance of these ions in the ion source, even if only one laser (laser I) is used. This can be done by allowing the alkali-metal ions to reside in the ion source for a longer time for additional reactions by delaying the application of the extraction pulse. The abundance of cationized quasimolecular ions in the ion source will increase if the gain in abundance due to the additional reactions exceeds the loss due to diffusion.

When KI was added to a gramicidin S/DHB mixture, the abundance of $\left[\mathrm{M}+\mathrm{K}^{+}\right.$ions of gramicidin $\mathrm{S}$ was found to be a function of the time between the firing of laser I (without laser II) and the application of the extraction pulse. KI has been added to control the alkali-metal ion content in the sample. As this delay time increases, the $[\mathrm{M}+\mathrm{K}]^{+}$signal increases until it reaches a maximum (Figure 4). The data points show considerable scatter, resulting from approximately $\pm 20 \%$ fluctuation from shot to shot. No attempt was therefore made to fit the data points to a curve. It is assumed, however, that only one maximum exists for a given molar ratio of gramicidin S to KI. Variation of the molar ratio of gramicidin $S$ to KI from 1:1 to $1: 3$ to 1:20, with gramicidin $S$ and DHB at a constant molar ratio of $1: 300$, results in a shift of the delay time at which such a curve has its maximum (DTM) toward shorter time; however, above a molar ratio of 20:1 between $\mathrm{KI}$ and gramicidin S, further increase in the content of KI in the sample has little effect on DTM. Significantly, it was observed that within the experimental precision, the abundance of the $[\mathrm{M}+\mathrm{K}]^{+}$ions at different DTMs was constant (the curves shown in Figure 4 were normalized to each maximum to minimize the systematic difference among different samples).

The observations above can be explained well by cation attachment in the gas phase. As the content of

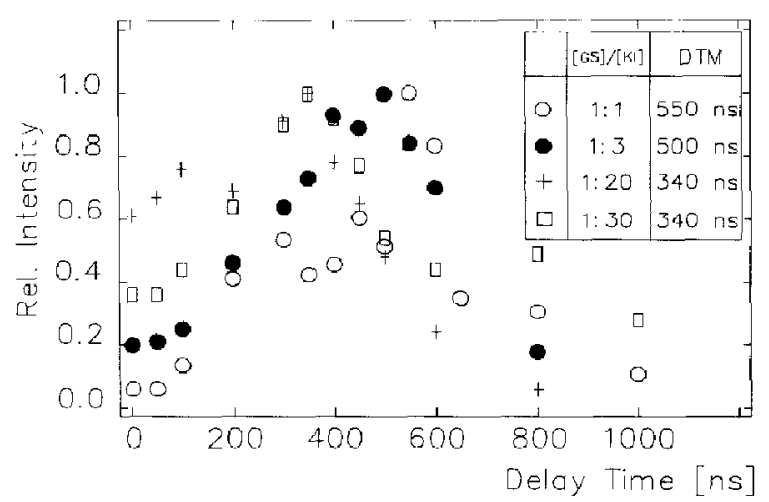

Figure 4. Effect of varying the delay time between laser pulse and ion extraction pulse on the abundance of $[\mathrm{M}+\mathrm{K}]^{+}$ions of gramicidin $S$ when gramicidin $S$ was mixed with KI in molar ratios of $1: 1,1: 3,1: 20$, and $1: 30$. The molar ratio between gramicidin $S$ and DHB was fixed at 1:300.
$\mathrm{KI}$ in the mixture increases, more $\mathrm{K}^{+}$ions are produced in the gas phase. If the number of gramicidin $\mathrm{S}$ molecules in the gas phase is constant, a higher abundance of $\mathrm{K}^{+}$ions will increase the cation attachment reaction rate and, consequently, shorten that time that it takes to reach the point at which the gain in the abundance of $[\mathrm{M}+\mathrm{K}]^{+}$due to additional reactions is offset by the loss due to diffusion. The constancy of ion abundance at different DTMs suggests that gramicidin $\mathrm{S}$ molecules in the gas phase were depleted by $\mathrm{K}^{+}$ ions. The fact that above a molar ratio of 20:1 between $\mathrm{KI}$ and gramicidin S, no further change in DTM was observed is probably due to saturation in the production of $\mathrm{K}^{+}$in the gas phase.

It is noteworthy that when the delay time is above $200 \mathrm{~ns}$, the abundance of the $\mathrm{K}^{+}$ions decreases sharply and monotonically as the delay time increases (Figure 5). When the delay time is shorter than $200 \mathrm{~ns}$, an even greater abundance of $\mathrm{K}^{+}$ions was measured, although the detector was saturated. These results indicate that no "delayed emission" of the $\mathrm{K}^{+}$ion takes place under the given conditions.

\section{Gas-Phase Protonation}

Predominantly $[\mathrm{M}+\mathrm{H}]^{+}$ions of gramicidin $S$ were produced when the crystalline rim instead of the center area of a gramicidin S/DHB sample was irradiated (Figure 6). The molar ratio between gramicidin $S$ and DHB was 1:300. This result is direct evidence that DHB can separate the analyte from salts by crystallizing with the analyte molecules on the rim while leaving the salts in the center area of the sample. This separation capability has been suggested to be responsible for the high tolerance of DHB for salt contamination in MALDI [7].

When the irradiance was doubled, thereby increasing the area from which ions and neutrals were desorbed, the abundance of the $[\mathrm{M}+\mathrm{H}]^{+}$ions decreased

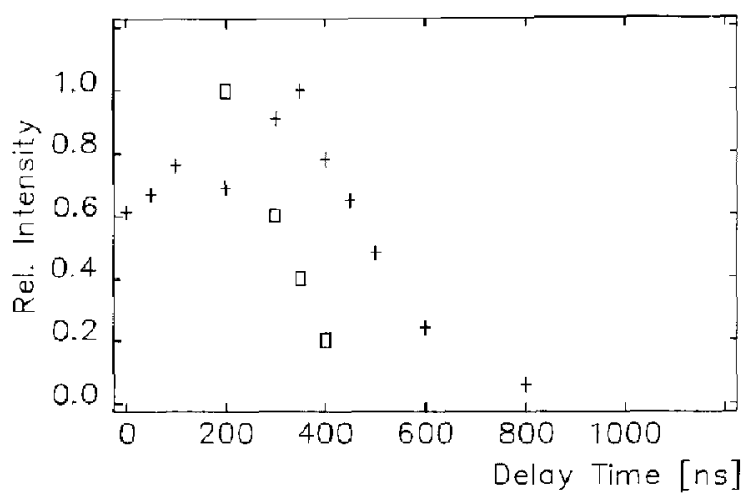

Figure 5. Abundances of the $(\square) K^{+}$and $(+)[M+K]^{+}$ions of gramicidin $S$ as the delay time between laser pulse and ion extraction pulse was varied. The molar ratio between gramicidin $\mathrm{S}, \mathrm{KI}$, and DHB was 1:20:300. At delay times below $200 \mathrm{~ns}$, the detector was saturated by the $\mathbf{K}^{+}$ions. 


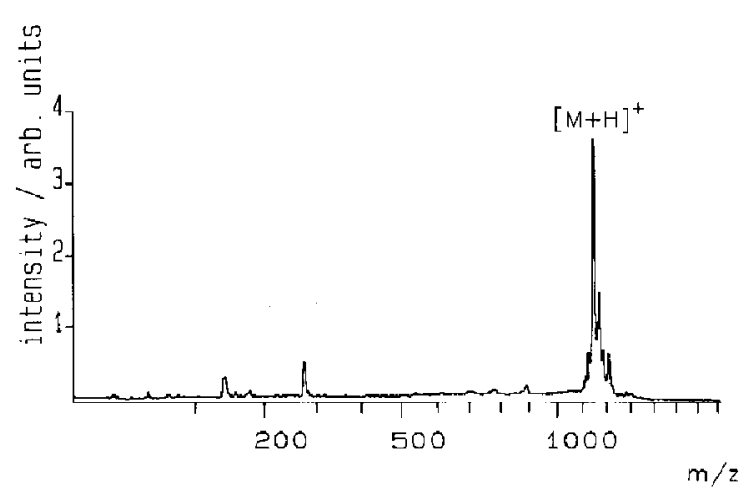

Figure 6. TOF mass spectrum of gramicidin 5 . Ions originated from the crystalline outer rim of the sample preparation. The molar ratio between gramicidin $S$ and DHB was 1:300; the delay between the desorption laser pulse and the ion extraction pulse was $400 \mathrm{~ns}$.

by $60 \%$, whereas that of the $[\mathrm{M}+\mathrm{Na}]^{+}$ion increased by $50 \%$. Accompanying the change, the abundance of the $\mathrm{Na}^{+}$ion increased by a factor of 100 , whereas the abundance of $\mathrm{M}^{+}$of $\mathrm{DHB}$ and its proton-attached homologue increased by a factor of 4 . Because gramicidin $\mathrm{S}$ molecules in the gas phase are the limiting reacting species, as discussed above, the reduction in the abundance of the $[\mathrm{M}+\mathrm{H}]^{+}$ions is most likely due to the competing cation attachment reaction. This result suggests that protonation of gramicidin $S$ is the result of ion-molecule reactions in the gas phase.

As in the case of the $[\mathrm{M}+\mathrm{K}]^{+}$ion of gramicidin $\mathrm{S}$, delayed extraction can also enhance the production of $[\mathrm{M}+\mathrm{H}]^{+}$ions for gramicidin $\mathrm{S}$. To examine the generality of this observation, HATI, melittin, and bovine insulin were also studied. In contrast to gramicidin S these compounds readily yield protonated quasimolecular ions by MALDI also from the center sample area. Figure 7 shows the mass spectrum of $\operatorname{HATII}(\mathrm{m} / \mathrm{z}$ 913.1). All of the compounds exhibit an enhancement in the abundance of $\left[\mathrm{M}+\mathrm{H}^{+}\right.$ions when ion extraction was delayed (Figure 8), with the DTM in the range $200-400$ ns. As in Figure 4, the apparent multi-

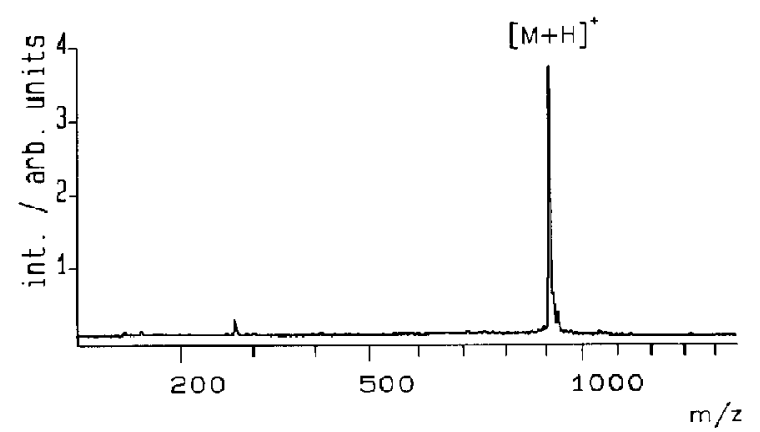

Figure 7. TOF mass spectrum of $\left[\mathrm{sar}^{1}, \mathrm{val}^{5}, \mathrm{ala}^{8}\right]$-human angiotensin II. The molar ratio between the analyte and DHB was 1:500. The delay between the desorption laser pulse and the ion extraction pulse was $300 \mathrm{~ns}$.

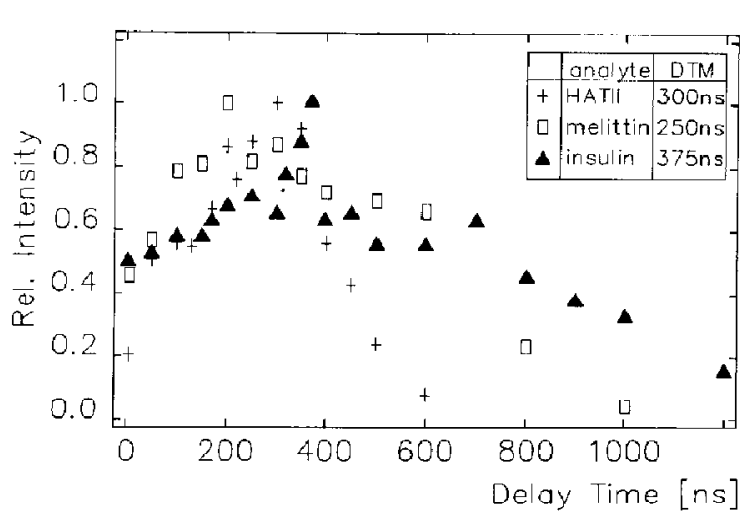

Figure 8. Intensities of the $\left[\mathbf{M}+\mathrm{H}^{+}\right.$peaks of HATII, melittin, and bovine insulin as functions of the delay time between the laser pulse and the ion extraction pulse. The molar ratios between the analytes and DHB were 1:500, 1:1000 and 1:20000, respectively.

ple peaks are probably due to the fluctuation of the data points $( \pm 20 \%)$; each curve is assumed to have only one maximum. These results suggest that by allowing the protonating agents (e.g., $\mathrm{M}^{+},[\mathrm{M}+\mathrm{H}]^{+}$, $[\mathrm{M}+2 \mathrm{H}]^{+}$of $\left.\mathrm{DHB}\right)$ to reside in the area near the sample surface, proton exchange can take place hundreds of nanoseconds after the desorption laser irradiation has ceased. Previous studies have suggested that the initial velocity of neutrals generated by MALDI is in the range of a few hundred meters per second [20, $21]$ and is relatively independent of mass. If the average speed of the peptide neutrals is assumed to be 500 $\mathrm{m} / \mathrm{s}$, then the maxima in the curves correspond to a distance $100-200 \mu \mathrm{m}$ from the sample surface. That ion-molecule reactions continue to take place at these distances is consistent with the results from the crossbeam experiment.

The DTMs in Figure 8 were found to be dependent

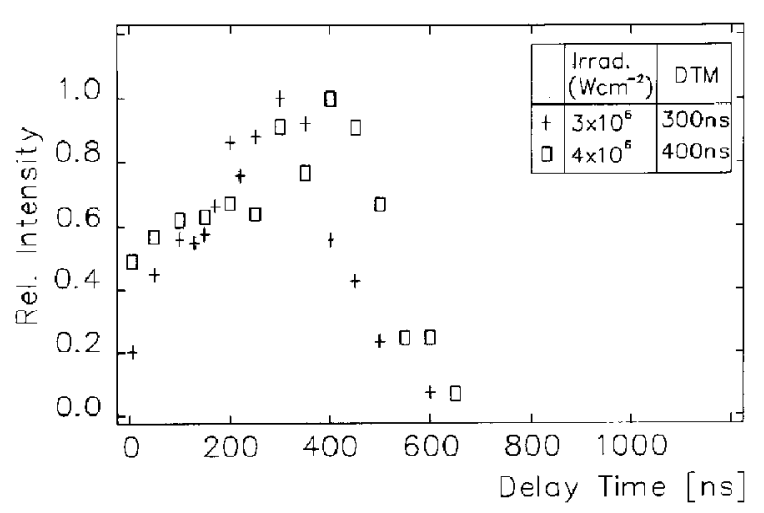

Figure 9. Intensity of the $[\mathrm{M}+\mathrm{H}]^{+}$peak of HATII as a function of the delay time between the laser pulse and the ion extraction pulse at two different irradiances: $(+) 3 \times 10^{6}$ and () $4 \times 10^{6} \mathrm{~W} / \mathrm{cm}^{2}$. The molar ratio between the analyte and DHB was 1:500. 
on the desorption laser irradiance. As shown in Figure 9 (the fluctuation of the data points is within $\pm 20 \%$ ), increasing the desorption laser irradiance from $3 \times 10^{6}$ to $4 \times 10^{6} \mathrm{~W} / \mathrm{cm}^{2}$ increased DTM by $100 \mathrm{~ns}$ for HATII. Because the increase in irradiance also resulted in an increase in the abundance of the $[\mathrm{M}+\mathrm{H}]^{+}$ions of HATII (not shown), this observation seems to reflect the fact that when there are more neutrals and protonating agents available, it takes longer to reach the point at which the loss due to diffusion is equal to the gain due to additional reactions.

\section{Conclusion}

By delaying the extraction of the ions produced under MALDI conditions, enhancement in the abundance of cation-attached or protonated quasimolecular ions was observed for all of the peptides studied, suggesting extensive ion-molecule reactions in the gas phase. This result is consistent with the postulate that matrix molecules undergo extensive photochemical reactions when enough photons are absorbed. Proton exchange hetween these photochemically activated matrix and analyte molecules leads to the formation of the [M + $\mathrm{H}]^{+}$ions. The fact that an enhancement lasts for a few hundred nanoseconds after the conclusion of the laser pulse shows that reaction cross sections for peptide protonation are large because they can occur a few hundred micrometers above the sample surface, where the density of proton-donating species and the neutral peptides has decreased significantly. This study also demonstrated that in a TOF system, it is possible to observe the cationization of peptide molecules if cations and peptides are desorbed separately by two lasers.

\section{Acknowledgments}

The authors thank Hanno Ehring for helpful discussions. Financial support from the Deutsche Forschungsgemeinschaft is appreciated. A research fellowship for $\mathrm{BHW}$ from the Alexander von Humboldt Foundation is also gratefully acknowledged.

\section{References}

1. Hillenkamp, F.; Karas, M.; Beavis, R. C.; Chait, B. Anal. Chem. 1991, 63, 1193A-1203A.

2. Karas, M.; Bahr, U.; Ingendoh, A.; Hillenkamp, F. Angezo. Chem. Int Ed. Engl. 1989, 28, 760-761.

3. Hillenkamp, F.; Karas, M.; Ingendoh, A.; Stahl, B. In Biological Mass Spectrometry; Burlingame, A. L; McCloskey, J. A., Eds.; Elsevier: Amsterdam, 1990; p 49.

4. Stahl, B.; Steup, M.; Karas, M.; Hillenkamp, F. Aral. Chem. 1991, 63, 1463-1466.

5. Mock, K. K.; Davey, M.; Cotrell, I. S. Biochem. Biophys. Res. Commun. 1991, 177, 644-651.

6. Deppe, A.; Bahr, U.; Karas, M.; Hillenkamp, F. Verhandlungen der Deutschen Physikalischen Gessellschaft; Frühjahrstagung: Hannover, 1992; p 1296.

7. Strupat, K.; Karas, M.; Hillenkamp, F. Int. J. Mass Spectrom. Ion. Processes 1991, 111, 89-102.

8. Strobel, F. H.; Solouki, T.; White, M. A.; Russell, D. H. J. Am. Soc. Mass Spectrom. 1991, 2, 91-94.

9. Hillenkamp, F.; Ehring, H. In Mass Spectrometry in the Biological Sciences: A Tutorial; Gross, M. L., Ed., Kluwer Academic Publishers: Dordrecht, The Netherlands, 1991; p 165.

10. van der Peyl, G. J. Q.; Isa, K.; Haverkamp; J; Kistemaker, P. G. Org. Mass Spectrom. 1981, 16, 416.

11. Stoll, R.; Röllgen, F. W. Z. Naturforsch. 1982, 9, 37a.

12. Cotter, R. J. Anal. Chim. Acta 1987, 195, 45-59.

13. Parker, C. D.; Hercules, D. M. Anal. Chem. 1986, 58, 25-34.

14. Karas, M.; Hillenkamp, F. In Ion Formation from Organic Solids: IFOS IV; Benninghoven, A., Ed:; Wiley: Chichester, 1987; p 103.

15. Speir, J. P.; Gorman, G. S.; Amster, I. J. Anat. Chem. 1991, 63, 65-69.

16. Ehring, H.; Karas, M.; Hillenkamp, F. Org. Mass. Spectrom. 1992, 27, 472-480.

17. Tabet, J.-C.; Cotter, R. J. Anal. Chem. 1984, 56, 1662-1667.

18. Keesee, R. G.; Castleman, A. W. J. Phys. Chem. Ref. Data 1986, 15, 1011-1071.

19. Kebarle, P. In Modern Aspects of Electrochemistry, Vol 9; Conway, B. E.; Bockris, J. O'M., Eds.; Plenum: New York, 1974; p 1.

20. Wang, B. H.; Dreisewerd, K.; Bahr, U.; Hillenkamp, F. Presented at the 2nd Workshop on Post Ionization Techniques in Surface Analysis, Pittsburgh, PA, May 15-17, 1991.

21. Huth-Fehre, T.; Becker, C. II. Rapid Commun. Mass Spectrom. 1991, $5,378-382$. 